Journal of Applied Mathematics and Stochastic Analysis, 16:3 (2003), 233-242.

Printed in the USA (C)2003 by North Atlantic Science Publishing Company

\title{
APPROXIMATE CONTROLLABILITY OF SEMILINEAR NEUTRAL SYSTEMS IN HILBERT SPACES
}

\author{
N. I. MAHMUDOV and S. ZORLU \\ Eastern Meditterranean University, Gazi Magusa \\ Mersin 10, Turkey \\ e-mail: nazim.mahmudov@emu.edu.tr, sonuc.zorlu@emu.edu.tr
}

(Received July 2002; Revised January 2003)

\begin{abstract}
The approximate controllability of semilinear neutral systems in Hilbert spaces is studied using the Schauder fixed point theorem. It is shown that the approximate controllability of the semilinear system under some conditions is implied by the approximate controllability of its linear part.

Keywords: Approximate Controllability, Semilinear Neutral Systems, The Schauder Fixed Point Theorem.
\end{abstract}

AMS (MOS) Subject Classification: 93B05, 34K40

\section{Introduction}

The theory of controllability of differential equations has been extensively studied in the literature. Zhou [9] studied the approximate controllability for a class of semilinear abstract equations. Naito [7] established the controllability for nonlinear Volterra integrodifferential systems. Chukwu and Lenhart [2] have studied controllability of nonlinear systems in abstract spaces. Hernández and Henriquez [4] obtained some existence results for neutral functional differential equations in Banach spaces by using semigroup theory and the Sadovski fixed point principle. Recently, Dauer and Balachandran [3], Balachandran and Sakthivel [1] studied exact controllability of neutral integrodifferential equations in Banach spaces using the Schaefer fixed point theorem. The purpose of this paper is to study the approximate controllability of semilinear neutral systems in Hilbert spaces by using the Schauder fixed point theorem.

Consider the following semilinear neutral system

$$
\begin{aligned}
\frac{d}{d t}[x(t)+g(t, x(t))] & =A x(t)+B u(t)+f(t, x(t)) \\
x(0) & =x_{0}, \quad t \in[0, T]
\end{aligned}
$$

where $A$ is the infinitesimal generator of an analytic semigroup of bounded linear operators $S(t)$ in Hilbert space $X, B: U \rightarrow X$ is a bounded linear operator, the control $u(\cdot) \in L_{2}([0, T], U)$ takes values in a Hilbert space $U$ and the state $x(\cdot)$ takes values in a space $X . f, g:[0, T] \times X \rightarrow X$ are continuous functions. 
The paper consists of 4 sections. Section 2 contains the definition of approximate controllability, the definition of mild solution and some basic assumptions on the data of the problem. Moreover, a formula for a control steering the initial state $x_{0}$ to a neighborhood of arbitrary final state is constructed. In Section 3, the main result on approximate controllability of semilinear neutral systems using the Schauder fixed point theorem is obtained. As an application of this result, the initial boundary-value problem is considered in Section 4.

\section{Preliminaries}

Definition 2.1: System (1.1) is said to be approximately controllable on the interval $[0, T]$ if

$$
\overline{\mathcal{R}\left(T, x_{0}\right)}=X
$$

where

$$
\mathcal{R}\left(T, x_{0}\right)=\left\{x\left(x_{0} ; u\right)(T): u(\cdot) \in L_{2}([0, T], U)\right\} .
$$

Definition 2.2: A function $x:[0, T] \rightarrow X$ is a mild solution of the abstract Cauchy problem (1.1) if $x(\cdot)$ is continuous on $[0, T]$; for each $0 \leq t<T$ the function $A S(t-s) g(s, x(s)), s \in[0, t)$, is integrable and

$$
\begin{aligned}
x(t) & =S(t)\left(x_{0}+g\left(0, x_{0}\right)\right)-g(t, x(t))-\int_{0}^{t} A S(t-s) g(s, x(s)) d s \\
& +\int_{0}^{t} S(t-s) f(s, x(s)) d s+\int_{0}^{t} S(t-s) B u(s) d s
\end{aligned}
$$

for $t \in[0, T]$.

The existence of mild solutions for the abstract Cauchy problem (1.1) follows from Theorem 2.1 and 2.2 of [4].

Recall that $A$ is the infinitesimal generator of an analytic semigroup of bounded linear operators $S(t)$ in $X$ such that

$$
\|S(t)\| \leq M_{1} \text { for some } M_{1} \geq 1
$$

At this point, it is possible to define the fractional power $(-A)^{\beta}$ of $(-A)$ for $0<\beta<1$ as a closed linear operator with domain $D\left((-A)^{\beta}\right)$. Furthermore the subspace $D\left((-A)^{\beta}\right)$ is dense in $X$ and the expression

$$
\|x\|_{\beta}=\left\|(-A)^{\beta} x\right\|
$$

defines a norm on $D\left((-A)^{\beta}\right)=X_{\beta}$ and there exists $C>0$ such that $\left\|(-A)^{-\beta}\right\| \leq C$ for $0 \leq \beta \leq 1$.

Lemma 2.1: [8] Let $A$ be the infinitesimal generator of an analytic semigroup $S(t)$. If $0 \in \rho(A)$ then for every $t>0$ the operator $(-A)^{\beta} S(t)$ is bounded and

$$
\left\|(-A)^{\beta} S(t)\right\| \leq C_{\beta} t^{-\beta} e^{-\delta t}, \delta>0 .
$$


We introduce two relevant operators and the basic assumption on these operators.

$$
\begin{aligned}
\Gamma_{0}^{T} & =\int_{0}^{T} S(T-s) B B^{*} S^{*}(T-s) d s \\
R\left(\alpha, \Gamma_{0}^{T}\right) & =\left(\alpha I+\Gamma_{0}^{T}\right)^{-1} .
\end{aligned}
$$

$(B A 1) \alpha R\left(\alpha, \Gamma_{0}^{T}\right) \rightarrow 0$ as $\alpha \rightarrow 0^{+}$in the strong operator topology.

It is known that the assumption (BA1) holds if and only if the linear system

$$
\begin{aligned}
& \dot{x}(t)=A x(t)+B u(t) \\
& x(0)=x_{0},
\end{aligned}
$$

is approximately controllable on $[0, T]$, see $[5,6]$.

The following theorem gives a formula for a control transferring the initial state $x_{0}$ to some neighborhood of $h$ at time $T$.

Theorem 2.1: For arbitrary $h \in X$, the control

$$
u(t)=B^{*} S^{*}(T-t) R\left(\alpha, \Gamma_{0}^{T}\right) p(x)
$$

where

$$
\begin{aligned}
p(x) & =h-S(T)\left[x_{0}+g\left(0, x_{0}\right)\right]+g(T, x(T)) \\
& -\int_{0}^{T} S(T-s) f(s, x(s)) d s+\int_{0}^{T} A S(T-s) g(s, x(s)) d s
\end{aligned}
$$

transfers the initial state $x_{0}$ to

$$
\begin{aligned}
x(T) & =h-\alpha\left(\alpha I+\Gamma_{0}^{T}\right)^{-1}\left(h-S(T)\left[x_{0}+g\left(0, x_{0}\right)\right]+g(T, x(T))\right. \\
& \left.-\int_{0}^{T} S(T-r) f(r, x(r)) d r+\int_{0}^{T} A S(T-r) g(r, x(r)) d r\right) .
\end{aligned}
$$

Proof: By substituting (2.3) in (2.1) and writing the obtained equation at $t=T$, one gets the following:

$$
\begin{aligned}
x(T) & =S(T)\left[x_{0}+g\left(0, x_{0}\right)\right]-g(T, x(T)) \\
& -\int_{0}^{T} A S(T-s) g(s, x(s)) d s+\int_{0}^{T} S(T-s) f(s, x(s)) d s \\
& +\int_{0}^{T} S(T-s) B B^{*} S^{*}(T-s)\left(\alpha I+\Gamma_{0}^{T}\right)^{-1} p(x) d s \\
& =S(T)\left[x_{0}+g\left(0, x_{0}\right)\right]-g(T, x(T)) \\
& -\int_{0}^{T} A S(T-s) g(s, x(s)) d s+\int_{0}^{T} S(T-s) f(s, x(s)) d s \\
& +\Gamma_{0}^{T}\left(\alpha I+\Gamma_{0}^{T}\right)^{-1} p(x) .
\end{aligned}
$$
$(2.4)$.

Using the identity $\Gamma_{0}^{T}\left(\alpha I+\Gamma_{0}^{T}\right)^{-1}=I-\alpha\left(\alpha I+\Gamma_{0}^{T}\right)^{-1}$ one can obtain equality 
The following notations are introduced for convenience:

$$
\begin{aligned}
K & =\max \left\{\left\|\Gamma_{0}^{t}\right\|: 0 \leq t \leq T\right\} \\
M & =\|B\|, \quad M_{0}=\max \left\{\left\|S(t) x_{0}\right\|: 0 \leq t \leq T\right\} \\
M_{2} & =\max \left\{\left\|S(t) g\left(0, x_{0}\right)\right\|: 0 \leq t \leq T\right\}
\end{aligned}
$$

We assume the following hypotheses:

(H1) The semigroup $S(t), t>0$ is analytic.

(H2) The function $f: I \times X \rightarrow X$ is continuous and there exists a constant $L>0$ such that

$$
\|f(t, x)\| \leq L \text { for all }(t, x) \in I \times X .
$$

(H3) The function $g$ is $X_{\beta}$ - valued, $(-A)^{\beta} g$ is continuous and there exists a constant $C_{1}>0$ such that

$$
\left\|(-A)^{\beta} g(t, x)\right\| \leq C_{1} \text { for all }(t, x) \in I \times X .
$$

(H4) For every $r>0$, the set of functions

$$
\left\{s \rightarrow(-A)^{\beta} g(s, x(s)): x \in C(0, T ; X), \sup _{t \in[0, T]}\|x(t)\| \leq r\right\}
$$

is equicontinuous on $[0, T]$.

\section{Approximate Controllability}

In this section, we prove the main result on approximate controllability of semilinear neutral systems. To do this, we first prove the existence of a fixed point of the operator $\mathfrak{F}$ defined below, using the Schauder fixed point theorem. Second, in Theorem 3 we show that under certain assumptions the approximate controllability of (1.1) is implied by the approximate controllability of the linear system (2.2).

Now, define the operator $\mathfrak{F}$ on $C([0, T], X)$ by means of

$$
\begin{aligned}
(\mathfrak{F} x)(t) & =S(t)\left[x_{0}+g\left(0, x_{0}\right)\right]-g(t, x(t))-\int_{0}^{t} A S(t-s) g(s, x(s)) d s \\
& +\int_{0}^{t} S(t-s) f(s, x(s)) d s+\int_{0}^{t} S(t-s) B u(s) d s,
\end{aligned}
$$

where

$$
\begin{aligned}
u(t) & =B^{*} S^{*}(T-t)\left(\alpha I+\Gamma_{0}^{T}\right)^{-1}\left(h-S(T)\left(x_{0}+g\left(0, x_{0}\right)\right)\right. \\
& +g(T, x(T))+\int_{0}^{T} A S(T-s) g(s, x(s)) d s \\
& \left.-\int_{0}^{T} S(T-s) f(s, x(s)) d s\right) .
\end{aligned}
$$


Introduce the set

$$
Y_{r}=\left\{x \in C([0, T], X): x(0)=x_{0},\|x\| \leq r\right\}
$$

where $r>0$.

Theorem 3.1: Assume hypotheses $(H 1),(H 2),(H 3),(H 4)$ and $(B A 1)$ are satisfied. Then for each $\alpha>0$, the operator $\mathfrak{F}$ has a fixed point.

Proof: The proof of the theorem is long and technical. Therefore it is convenient to divide it into several steps.

Step 1 : For all $\alpha>0$, there exists $r=r(\alpha)$ such that $\mathfrak{F}$ maps $Y_{r}$ into $Y_{r}$.

Using the fractional powers of $(-A)$ the operator (3.1) can be rewritten as follows.

$$
\begin{aligned}
(\mathfrak{F} x)(t) & =S(t)\left(x_{0}+g\left(0, x_{0}\right)\right)-(-A)^{-\beta}(-A)^{\beta} g(t, x(t)) \\
& -\int_{0}^{t}(-A)^{1-\beta} S(t-s)(-A)^{\beta} g(s, x(s)) d s \\
& +\int_{0}^{t} S(t-s) f(s, x(s)) d s+\int_{0}^{t} S(t-s) B u(s) d s
\end{aligned}
$$

where

$$
\begin{aligned}
u(t) & =B^{*} S^{*}(T-t)\left(\alpha I+\Gamma_{0}^{T}\right)^{-1}\left(h-S(T)\left(x_{0}+g\left(0, x_{0}\right)\right)\right. \\
& +(-A)^{-\beta}(-A)^{\beta} g(T, x(T))-\int_{0}^{T} S(T-s) f(s, x(s)) d s \\
& \left.+\int_{0}^{T}(-A)^{1-\beta} S(T-s)(-A)^{\beta} g(s, x(s)) d s\right) .
\end{aligned}
$$

Taking norm on (3.3) and (3.4), yields

$$
\begin{aligned}
\|(\mathfrak{F} x)(t)\| & \leq\left\|S(t) x_{0}\right\|+\|S(t)\|\left\|g\left(0, x_{0}\right)\right\|+\left\|(-A)^{-\beta}(-A)^{\beta} g(t, x(t))\right\| \\
& +\int_{0}^{t}\left\|(-A)^{1-\beta} S(t-s)\right\|\left\|(-A)^{\beta} g(s, x(s))\right\| d s \\
& +\int_{0}^{t}\|S(t-s)\|\|f(s, x(s))\| d s+\int_{0}^{t}\|S(t-s)\|\|B\|\|u(s)\| d s \\
& \leq M_{0}+M_{2}+C C_{1}+\int_{0}^{t} \frac{C_{\beta} C_{1}}{(t-s)^{1-\beta}} d s+M_{1} L T+M M_{1} \sqrt{T}\|u\| \\
& \leq M_{0}+M_{2}+C C_{1}+\frac{C_{1} C_{\beta} T^{\beta}}{\beta}+M_{1} L T+M M_{1} \sqrt{T}\|u\|
\end{aligned}
$$

and

$$
\begin{aligned}
\|u(t)\| & \leq \frac{1}{\alpha} M M_{1}\left(\|h\|+M_{0}+M_{2}+C C_{1}+\int_{0}^{T} \frac{C_{1} C_{\beta}}{(T-s)^{1-\beta}} d s+M_{1} T L\right) \\
& \leq \frac{1}{\alpha} M M_{1}\left(\|h\|+M_{0}+M_{2}+C C_{1}+\frac{C_{1} C_{\beta} T^{\beta}}{\beta}+M_{1} T L\right) .
\end{aligned}
$$


The last two inequalities (3.5) and (3.6) imply that for large enough $r>0$ the following inequality holds

$$
\|(\mathfrak{F} x)(t)\| \leq r
$$

That is $(\mathfrak{F} x) \in Y_{r}$. Therefore $\mathfrak{F}$ maps $Y_{r}$ into itself.

Step 2. $V(t)=\left\{(\mathfrak{F} x)(t): x \in Y_{r}\right\}$ is relatively compact for arbitrary $t \in[0, T]$.

Since $V(0)=\left\{x_{0}\right\}$, the case where $t=0$ is trivial. So let $t, 0<t \leq T$, be a fixed point and let $\eta$ be a given real number satisfying $0<\eta<t$. Define

$$
\left(\mathfrak{F}_{1}^{\eta} x\right)(t)=S(\eta)\left(\mathfrak{F}_{1} x\right)(t-\eta)
$$

where

$$
\begin{aligned}
\left(\mathfrak{F}_{1} x\right)(t-\eta) & =S(t-\eta)\left(x_{0}+g\left(0, x_{0}\right)\right) \\
& +\int_{0}^{t-\eta} S(t-s-\eta) B B^{*} S^{*}(T-s)\left(\alpha I+\Gamma_{0}^{T}\right)^{-1} p(x) d s \\
& -\int_{0}^{t-\eta}(-A)^{1-\beta} S(t-s-\eta)(-A)^{\beta} g(s, x(s)) d s \\
& +\int_{0}^{t-\eta} S(t-s-\eta) f(s, x(s)) d s .
\end{aligned}
$$

Since $S(t)$ is analytic and $\mathfrak{F}_{1}$ is bounded on $Y_{r}$, by assumption $(H 4)$, the set

$$
V_{\eta}(t)=\left\{\left(\mathfrak{F}_{1}^{\eta} x\right)(t)+g(t, x(t)): x \in Y_{r}\right\}
$$

is relatively compact set in $X$. That is, a finite set $\left\{y_{i}, 1 \leq i \leq m\right\}$ in $X$ exists such that

$$
V_{\eta}(t) \subset \bigcup_{i=0}^{m} N\left(y_{i}, \frac{\varepsilon}{2}\right),
$$

where $N\left(y_{i}, \frac{\varepsilon}{2}\right)$ is an open ball in $X$ with the center at $y_{i}$ and radius $\frac{\varepsilon}{2}$.

On the other hand,

$$
\begin{aligned}
\left\|\left(\mathfrak{F}_{1} x\right)(t)-\left(\mathfrak{F}_{1}^{\eta} x\right)(t)\right\| & =\| \int_{t-\eta}^{t} S(t-s) B B^{*} S^{*}(T-s)\left(\alpha I+\Gamma_{0}^{T}\right)^{-1} p(x) d s \\
& -\int_{t-\eta}^{t}(-A)^{1-\beta} S(t-s)(-A)^{\beta} g(s, x(s)) d s \\
& +\int_{t-\eta}^{t} S(t-s) f(s, x(s)) d s \| \\
& \leq \frac{1}{\alpha} M^{2} M_{1}^{2} P \eta+\frac{C_{1} C_{\beta} \eta^{\beta}}{\beta}+M_{1} L \eta \\
& \leq \frac{\varepsilon}{2} .
\end{aligned}
$$

Consequently,

$$
V(t) \subset \bigcup_{i=0}^{m} N\left(y_{i}, \varepsilon\right) .
$$

Hence, $V(t)$ is relatively compact in $X$ for each $t \in[0, T]$. 
Step 3. $V=\left\{\mathfrak{F} x: x \in Y_{r}\right\}$ is equicontinuous on $t \in[0, T]$.

For $0<t_{1}-\varepsilon<t_{1}<t_{2}<T$,

$$
\begin{aligned}
& \left(\mathfrak{F}_{1} x\right)\left(t_{1}\right)-\left(\mathfrak{F}_{1} x\right)\left(t_{2}\right)=\left(S\left(t_{1}-s\right)-S\left(t_{2}-s\right)\right)\left(x_{0}+g\left(0, x_{0}\right)\right) \\
& +\int_{0}^{t_{1}}\left[S\left(t_{1}-s\right)-S\left(t_{2}-s\right)\right] f(s, x(s)) d s-\int_{t_{1}}^{t_{2}} S\left(t_{2}-s\right) f(s, x(s)) d s \\
& +\int_{0}^{t_{1}-\varepsilon}(-A)^{1-\beta} S\left(t_{1}-\varepsilon-s\right)\left(I-S\left(t_{2}-t_{1}\right)\right) S(\varepsilon)(-A)^{\beta} g(s, x(s)) d s \\
& +\int_{t_{1}-\varepsilon}^{t_{1}}\left(I-S\left(t_{2}-t_{1}\right)\right)(-A)^{1-\beta} S\left(t_{1}-s\right)(-A)^{\beta} g(s, x(s)) d s \\
& -\int_{t_{1}}^{t_{2}}(-A)^{1-\beta} S\left(t_{2}-s\right)(-A)^{\beta} g(s, x(s)) d s \\
& +\int_{0}^{t_{1}}\left(S\left(t_{1}-s\right)-S\left(t_{2}-s\right)\right) B u(s) d s-\int_{t_{1}}^{t_{2}} S\left(t_{2}-s\right) B u(s) d s .
\end{aligned}
$$

Taking norm on (3.7) and using hypotheses $(H 2),(H 3)$ and Lemma 2.1,

$$
\begin{aligned}
\|\left(\mathfrak{F}_{1} x\right)\left(t_{1}\right) & -\left(\mathfrak{F}_{1} x\right)\left(t_{2}\right)\|\leq\| S\left(t_{1}-s\right)-S\left(t_{2}-s\right) \|\left(\left\|x_{0}\right\|+M_{3}\right) \\
& +L \int_{0}^{t}\left\|S\left(t_{1}-s\right)-S\left(t_{2}-s\right)\right\| d s+\left(t_{2}-t_{1}\right) M_{1} L \\
& +\frac{\left(t_{1}-\varepsilon\right)^{\beta}}{\beta} M_{1} C_{1}\left\|I-S\left(t_{2}-t_{1}\right)\right\| \\
& +\frac{\varepsilon^{\beta}}{\beta} C_{1}\left\|I-S\left(t_{2}-t_{1}\right)\right\|+\frac{C_{1}\left(t_{2}-t_{1}\right)^{\beta}}{\beta} \\
& +M \int_{0}^{t}\left\|S\left(t_{1}-s\right)-S\left(t_{2}-s\right)\right\|\|u(s)\| d s \\
& +M M_{1}\|u\| \sqrt{t_{2}-t_{1}} \\
& =I_{1}+I_{2}+I_{3}+I_{4}+I_{5}+I_{6}+I_{7}+I_{8} .
\end{aligned}
$$

The right hand side of (3.8) does not depend on particular choices of $x$. It is clear that $I_{3} \rightarrow 0, I_{6} \rightarrow 0$ and $I_{8} \rightarrow 0$ as $t_{2}-t_{1} \rightarrow 0$. Since the semigroup $S(t), t>0$ is analytic

$$
\left\|S\left(t_{1}-s\right)-S\left(t_{2}-s\right)\right\| \rightarrow 0
$$

as $t_{1}-t_{2} \rightarrow 0$ for arbitrary $t, s$ such that $t-s>0$. By Lebesque dominated convergence theorem $I_{2} \rightarrow 0$ and $I_{7} \rightarrow 0$ as $t_{2}-t_{1} \rightarrow 0$. It is obvious that, $I_{4} \rightarrow 0, I_{5} \rightarrow 0$ as $t_{2}-t_{1} \rightarrow 0$. Hence $V$ is equicontinuous and the equicontinuity of $g$ follows from $(H 4)$.

Note that we only considered the case when $0<t_{1}<t_{2}$, since the other case $0<t_{2}<t_{1}$ is similar. Therefore $\mathfrak{F}\left[Y_{r}\right]$ is equicontinuous and bounded. By the Arzela Ascoli theorem, $\mathfrak{F}\left[Y_{r}\right]$ is relatively compact in $C([0, T], X)$.

Step 4. $\mathfrak{F}$ is continuous on $C([0, T], X)$.

In order to apply the Schauder fixed point theorem it remains to show the continuity of $\mathfrak{F}$ on $C([0, T], X)$. Let $\left\{y_{n}\right\} \subset C([0, T], X)$ with $y_{n} \rightarrow y$ in $C([0, T], X)$. Since

$$
\begin{aligned}
f\left(s, y^{n}(s)\right) & \rightarrow f(s, y(s)), \quad \text { for each } s \in[0, T] \\
(-A)^{\beta} g\left(s, y^{n}(s)\right) & \rightarrow(-A)^{\beta} g(s, y(s)), \text { for each } s \in[0, T],
\end{aligned}
$$


and

$$
\begin{aligned}
\left\|\left(\mathfrak{F} y^{n}\right)(t)-(\mathfrak{F} y)(t)\right\| & \leq C\left\|(-A)^{\beta}\left(g\left(t, y^{n}(t)\right)-g(t, y(t))\right)\right\| \\
& +M M_{1} \sqrt{T}\left(\int_{0}^{t}\left\|u^{n}(s)-u(s)\right\|^{2} d s\right)^{1 / 2} \\
& +\frac{t^{\beta}}{\beta}\left\|(-A)^{\beta}\left(g\left(t, y^{n}(t)\right)-g(t, y(t))\right)\right\| \\
& +M_{1} \int_{0}^{t}\left\|f\left(s, y^{n}(s)\right)-f(s, y(s))\right\| d s,
\end{aligned}
$$

the Lebesque dominated convergence theorem implies that

$$
\sup _{0 \leq t \leq T}\left\|\left(\mathfrak{F} y^{n}\right)(t)-(\mathfrak{F} y)(t)\right\| \rightarrow 0,
$$

which shows the continuity of $\mathfrak{F}$. Thus all the conditions of the Schauder fixed point theorem are satisfied, and consequently the operator $\mathfrak{F}$ has a fixed point in $C([0, T], X)$.

Theorem 3.2: Assume hypotheses $(H 1),(H 2),(H 3)$ and $(B A 1)$ are satisfied. Then the system (1.1) is approximately controllable on $[0, T]$.

Proof: Let $x^{\alpha}(\cdot)$ be a fixed point of $\mathfrak{F}$ in $Y_{r}$. By Theorem 2.1, any fixed point of $\mathfrak{F}$ is a mild solution of (1.1) on $[0, T]$ under the control

$$
u^{\alpha}(t)=B^{*} S^{*}(T-t) R\left(\alpha, \Gamma_{0}^{T}\right) p\left(x^{\alpha}\right)
$$

and satisfies

$$
\begin{aligned}
x^{\alpha}(T) & =h-\alpha R\left(\alpha, \Gamma_{0}^{T}\right)\left\{h-S(T)\left[x_{0}+g\left(0, x_{0}\right)\right]+g(T, x(T))\right. \\
& \left.+\int_{0}^{T} A S(T-s) g\left(s, x^{\alpha}(s)\right) d s-\int_{0}^{T} S(T-s) f\left(s, x^{\alpha}(s)\right) d s\right\} .
\end{aligned}
$$

By $(H 2)$ and $(H 3)$,

$$
\begin{gathered}
\int_{0}^{T}\left\|f\left(s, x^{\alpha}(s)\right)\right\|^{2} d s \leq L^{2} T \\
\int_{0}^{T}\left\|(-A)^{\beta} g\left(s, x^{\alpha}(s)\right)\right\|^{2} d s \leq C_{1}^{2} T .
\end{gathered}
$$

Consequently, the sequences $\left\{f\left(s, x^{\alpha}(s)\right)\right\}$ and $\left\{(-A)^{\beta} g\left(s, x^{\alpha}(s)\right)\right\}$ are bounded in $L_{2}([0, T], X)$. Thus there are subsequences, still denoted by $\left\{f\left(s, x^{\alpha}(s)\right)\right\}$ and $\left\{(-A)^{\beta} g\left(s, x^{\alpha}(s)\right)\right\}$, that converge weakly to say $f(s)$ and $g(s)$ in $L_{2}([0, T], X)$, respectively. Define

$$
\begin{aligned}
q & =h-S(T)\left[x_{0}+g\left(0, x_{0}\right)\right]+g(T, x(T)) \\
& +\int_{0}^{T}(-A)^{-\beta} S(T-s) g(s) d s-\int_{0}^{T} S(T-s) f(s) d s .
\end{aligned}
$$


It follows that

$$
\begin{aligned}
\left\|p\left(x^{\alpha}\right)-q\right\| & \leq\left\|\int_{0}^{T} S(T-s)\left(f\left(s, x^{\alpha}(s)\right)-f(s)\right) d s\right\| \\
& +\left\|\int_{0}^{T}(-A)^{-\beta} S(T-s)\left((-A)^{\beta} g\left(s, x^{\alpha}(s)\right)-g(s)\right) d s\right\| .
\end{aligned}
$$

By the compactness of the operators $k(t) \rightarrow \int_{0}^{t} S(t-s) k(s) d s: L_{2}([0, T], X) \rightarrow$ $C([0, T], X)$ and $k(t) \rightarrow \int_{0}^{t}(-A)^{-\beta} S(t-s) k(s) d s: L_{2}([0, T], X) \rightarrow C([0, T], X)$, the right hand side of (3.10) tends to zero as $\alpha \rightarrow 0^{+}$.

By (3.9),

$$
\begin{aligned}
\left\|x^{\alpha}(T)-h\right\| & =\left\|\alpha R\left(\alpha, \Gamma_{0}^{T}\right) p\left(x^{\alpha}\right)\right\| \\
& =\left\|\alpha R\left(\alpha, \Gamma_{0}^{T}\right)\left(p\left(x^{\alpha}\right)-q+q\right)\right\| \\
& \leq\left\|\alpha R\left(\alpha, \Gamma_{0}^{T}\right) q\right\|+\left\|\alpha R\left(\alpha, \Gamma_{0}^{T}\right)\left(p\left(x^{\alpha}\right)-q\right)\right\| \\
& \leq\left\|\alpha R\left(\alpha, \Gamma_{0}^{T}\right) q\right\|+\left\|\alpha R\left(\alpha, \Gamma_{0}^{T}\right)\right\|\left\|p\left(x^{\alpha}\right)-q\right\| \\
& \leq\left\|\alpha R\left(\alpha, \Gamma_{0}^{T}\right) q\right\|+\left\|p\left(x^{\alpha}\right)-q\right\|,
\end{aligned}
$$

where (3.11) tends to zero as $\alpha \rightarrow 0^{+}$by the estimation (3.10) and the assumption $(B A 1)$. This proves the approximate controllability of system (1.1).

Note that compactness of the operators $k(\cdot) \rightarrow \int_{0}^{\cdot} S(\cdot-s) k(s) d s: L_{2}([0, T], X) \rightarrow$ $C([0, T], X)$ and $k(\cdot) \rightarrow \int_{0}^{\cdot}(-A)^{-\beta} S(\cdot-s) k(s) d s: L_{2}([0, T], X) \rightarrow C([0, T], X)$ are essential in the above proof.

\section{Applications}

In this section, we illustrate the obtained result. Let $X=L_{2}[0, \pi]$ and $A$ be defined as follows

$$
A z=z^{\prime \prime}
$$

with domain

$$
D(A)=\left\{f(\cdot) \in L_{2}[0, \pi]: f^{\prime \prime} \in L_{2}[0, \pi], f(0)=f(\pi)=0\right\} .
$$

Recall that $A$ is the infinitesimal generator of a strongly continuous semigroup $S(t), t>$ 0 , on $X$ which is analytic and self-adjoint, the eigenvalues are $-n^{2}, n \in N$, with corresponding normalized eigenvectors $z_{n}(\xi):=(2 / \pi)^{1 / 2} \sin (n \xi)$. Moreover the following hold:

(a) $\left\{z_{n}: n \in N\right\}$ is an orthonormal basis of $X$.

(b) If $f \in D(A)$ then $A(f)=-\sum_{n=1}^{\infty} n^{2}\left\langle f, z_{n}\right\rangle z_{n}$.

(c) For $f \in X,(-A)^{-1 / 2} f=\sum_{n=1}^{\infty} \frac{1}{n}\left\langle f, z_{n}\right\rangle z_{n}$.

(d) The operator $(-A)^{1 / 2}$ is given as $(-A)^{1 / 2} f=\sum_{n=1}^{\infty} n\left\langle f, z_{n}\right\rangle z_{n}$ on the space $D\left((-A)^{1 / 2}\right)=\left\{f \in X: \sum_{n=1}^{\infty} n\left\langle f, z_{n}\right\rangle z_{n} \in X\right\}$. 
Consider the neutral system

$$
\begin{aligned}
\frac{d}{d t}[x(t, \xi)+q(t, x(t, \xi))] & =\frac{\partial^{2}}{\partial \xi^{2}} x(t, \xi) \\
& +p(t, x(t, \xi))+B u(t, \xi), \\
x(t, 0) & =x(t, \pi)=0, t \geq 0, \\
x(0, \xi) & =\varphi(\xi), 0 \leq \xi \leq \pi,
\end{aligned}
$$

where $p:[0, T] \times R \rightarrow R, q:[0, T] \times R \rightarrow R$ are continuous functions, the control $u \in U=L_{2}([0, T] \times[0, \pi])$ and $B$ is a linear bounded operator from $U$ to $X$.

To write the initial-boundary value problem $(4.1)-(4.3)$ in the abstract form we assume the following:

(i) The substitution operator $f: R \times X \rightarrow X$ defined by $f(t, x(t))=p(t, x(t, \xi))$ is continuous and there exists $L>0$ such that $\|f(t, x)\| \leq L,(t, x) \in R \times X$.

(ii) The substitution operator $g: R \times X \rightarrow X$ defined by $g(t, x(t))=q(t, x(t, \xi))$ is such that $(-A)^{1 / 2} g$ is completely continuous on $R \times X$ and there exists $L>0$ such that $\left\|(-A)^{1 / 2} g(t, x)\right\| \leq L,(t, x) \in R \times X$.

Thus the problem $(4.1)-(4.3)$ can be written in the abstract form

$$
\begin{aligned}
\frac{d}{d t}(x(t)+g(t, x(t))) & =A x(t)+f(t, x(t))+B u(t) \\
x(0) & =x_{0}, \quad t \in[0, T]
\end{aligned}
$$

and all the conditions of the Theorem 3 are satisfied.This gives the approximate controllability of the neutral system $(4.1)-(4.3)$.

\section{References}

[1] Balachandran, K. and Sakthivel, R., Existence of solutions for neutral functional integrodifferential equations in Banach spaces. Proc. Indian Acad. Sci. Math. Sci., 109, (1999), 325-332.

[2] Chukwu, E. N. and Lenhart, S. M., Controllability questions for nonlinear systems in abstract spaces, Journ. Optim. Theory and Appl., 68,(1991), 437-462.

[3] Dauer, J. P. and Balachandran, K., Existence of solutions of nonlinear neutral integrodifferential equations in Banach spaces. Journ. Math. Anal. Appl., 251:1, (2000), 93-105.

[4] Hernández, E. and Henriquez, H. R, Existence results for partial neutral equations with unbounded delay, Journ. Math. Anal. Appl., 221,(1998), 452-475.

[5] Mahmudov, N. I., On Controllabiltiy of linear stochastic systems, IEEE Trans. Automat. Control, 46:5, (2001).

[6] Mahmudov, N. I., On Controllabiltiy of linear stochastic systems in Hilbert spaces, Journ. Math. Anal. Appl., 259:1, (2001), 64-82.

[7] Naito, K., On controllability for a nonlinear Volterra equation, Nonlinear Analysis: Theory, Methods and Applications, 18, (1992), 99-108.

[8] Pazy, A., Semigroups of Linear Operators and Applications to Partial Differential Equations, Applied Mathematical Sciences, 44. Springer-Verlag, NewYork-Berlin, 1983.

[9] Zhou, X., Exact controllability of semilinear evolution systems and its applications, Journ. Optim. Theory and Appl., 107 (2000), 415-432. 


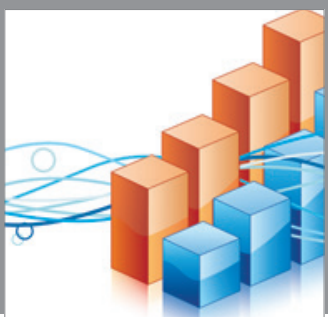

Advances in

Operations Research

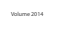

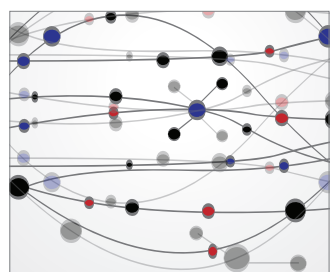

\section{The Scientific} World Journal
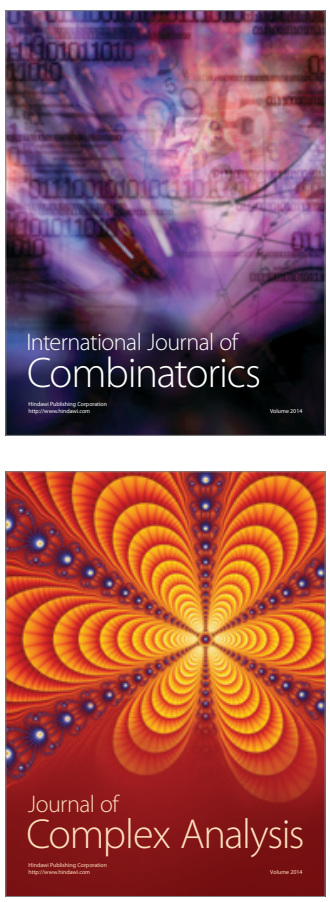

International Journal of

Mathematics and

Mathematical

Sciences
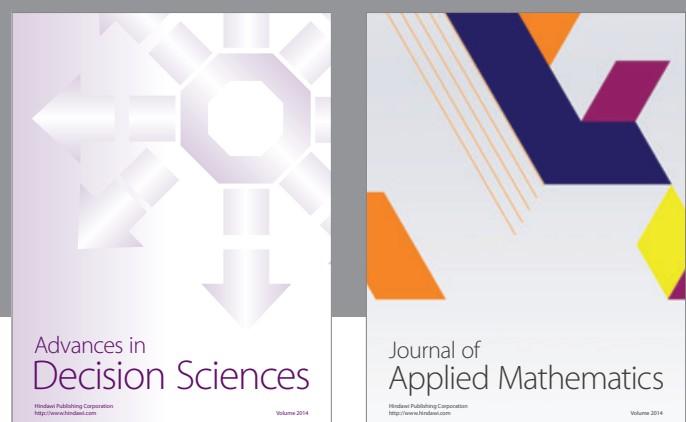

Journal of

Applied Mathematics
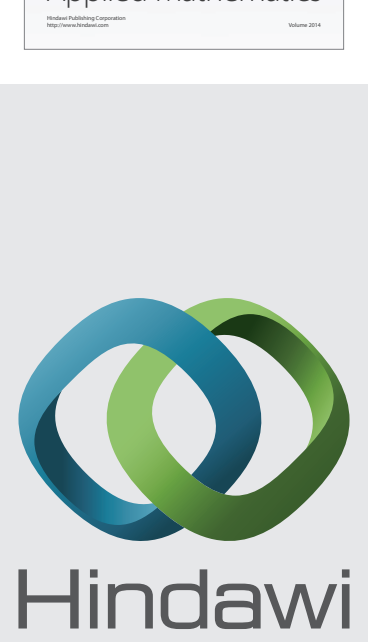

Submit your manuscripts at http://www.hindawi.com
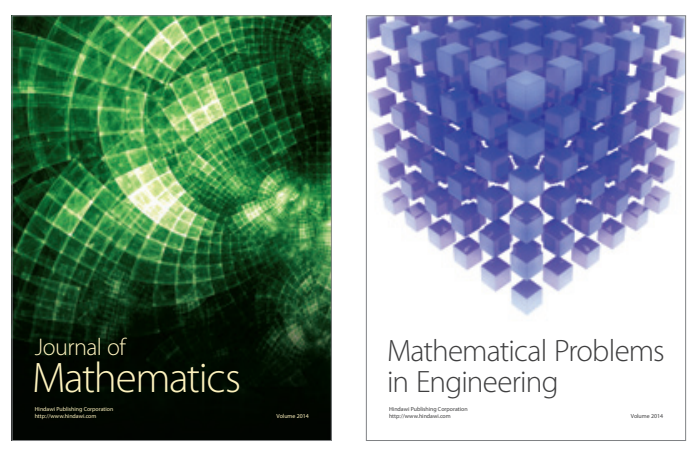

Mathematical Problems in Engineering
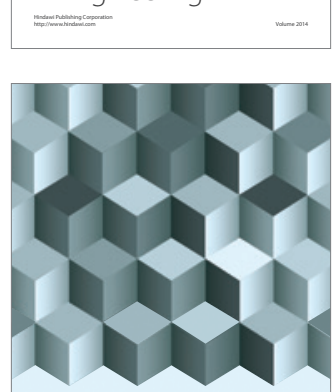

Journal of

Function Spaces
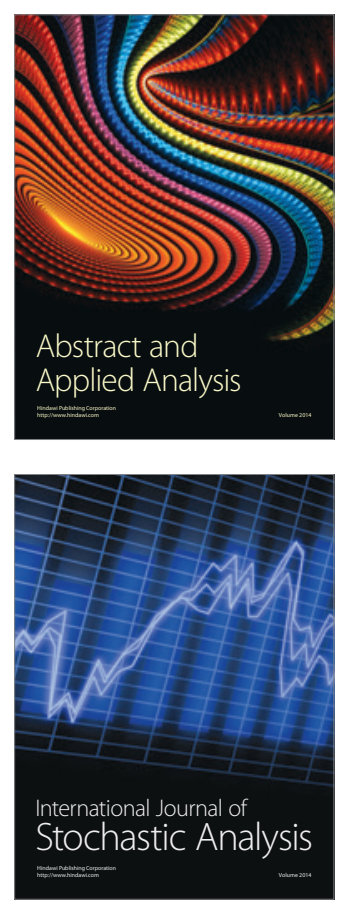

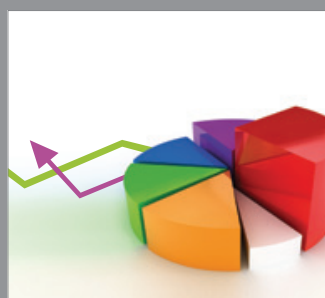

ournal of

Probability and Statistics

Promensencen
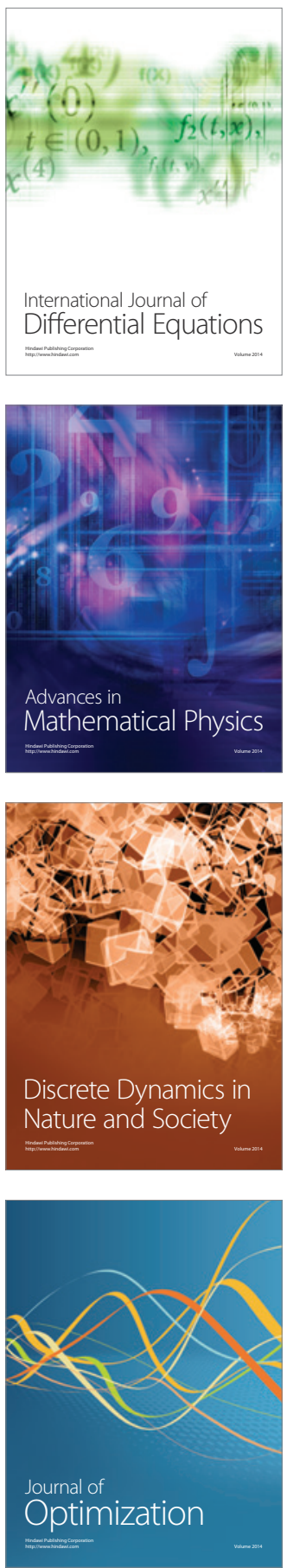